\title{
Fusion in conformal field theory as the tensor product of the symmetry algebra
}

\author{
Matthias Gaberdiel * \\ Department of Applied Mathematics and Theoretical Physics \\ University of Cambridge, Silver Street \\ Cambridge, CB3 9EW, U. K.
}

July 1993

\begin{abstract}
Following a recent proposal of Richard Borcherds to regard fusion as the ring-like tensor product of modules of a quantum ring, a generalization of rings and vertex operators, we define fusion as a certain quotient of the (vector space) tensor product of representations of the symmetry algebra $\mathcal{A}$. We prove that this tensor product is associative and symmetric up to equivalence. We also determine explicitly the action of $\mathcal{A}$ on it, under which the central extension is preserved.

Having given a precise meaning to fusion, determining the fusion rules is now a well-posed algebraic problem, namely to decompose the tensor product into irreducible representations. We demonstrate how to solve it for the case of the WZW- and the minimal models and recover thereby the well-known fusion rules.

The action of the symmetry algebra on the tensor product is given in terms of a comultiplication. We calculate the $R$-matrix of this comultiplication and find that it is triangular. This seems to shed some new light on the possible rôle of the quantum group in conformal field theory.
\end{abstract}

*e-mail: mg10004@amtp.cam.ac.uk 


\section{Introduction}

The notion of fusion plays a very important rôle in conformal field theory, however, as far as the definition of fusion is concerned the state of affairs is not entirely satisfactory. There exist on the one hand the physicists' definition of fusion in terms of nonvanishing three-point-functions, which has the advantage of being easily calculable, which however is conceptually not very appealing. On the other hand there exist different definitions in the mathematical literature, e. g. in terms of holomorphic embeddings [13], more algebraically by Feigin and Fuchs [4] (for the minimal models) and by Kazhdan and Lusztig [9] (for the Kac-Moody algebras), or in the spirit of algebraic quantum field theory [14], however, these definitions tend to be rather abstract and it seems quite difficult to perform any calculations in these setups. It seems therefore quite interesting to give a precise mathematical definition of fusion, which is at the same time sufficiently explicit to allow for example a simple derivation of the fusion rules.

The present paper was motivated and inspired by the recent proposal of Richard Borcherds [3] to introduce the concept of a quantum ring, a generalization of rings and vertex algebras for which the holomorphic fields are a natural example, to give a precise meaning to fusion. From this point of view conformal field theory is just the representation theory of this quantum ring and fusion corresponds to taking the canonical ring-like tensor product of modules, namely the usual vector space tensor product quotiented by the relations which guarantee that the action of the quantum ring on both modules is the same. Richard Borcherds showed that the corresponding tensor category is isomorphic to the tensor category of Kazhdan and Lusztig, which is believed to coincide with the physicists' definition of fusion.

To express this approach to conformal field theory in more physical terms we have to relate the quantum ring of holomorphic fields to the symmetry algebra, i. e. to the mode expansion of the holomorphic fields. We use the methods of [8] to analyze conformal field theory in detail and derive explicitly the action of the symmetry algebra on products of vertex operators. This action can be interpreted as a comultiplication of the symmetry algebra (depending on two parameters, namely the insertion points of the vertex operators), under which the central charge is preserved, and can thus be used to define a (vector space) tensor product of modules of the symmetry algebra. (This comultiplication is a generalization of the formula given in [12].)

To derive the comultiplication formula we have to use the operator product expansion of the holomorphic field with one of the vertex operators. The formula 
we obtain is thus quite asymmetric, as we have treated the two vertex operators differently. If we used the operator product expansion with the other vertex operator, we would get a different formula, which, on the underlying conformal field theory, must agree with the previous expression. We cannot impose this equality on the level of the algebra, as the two formulae are only formally well-defined power series, however, we can implement it on the tensor product of representations. This leads us to define the "true" tensor product of conformal field theory to be the quotient of the original (vector space) tensor product by all relations which arise in this way. By quotienting out this subspace we guarantee that the action of the holomorphic fields on both vertex operators is the same; our tensor product corresponds thus exactly to the ring-like tensor product of quantum ring modules.

The next step is to prove general properties of this tensor product. Firstly, we determine the dependence of the tensor product on the two parameters and show that all different choices are equivalent. We prove that the comultiplication is coassociative and thus that the tensor product is associative up to equivalence. We then calculate the $R$-matrix of the comultiplication. It turns out that it is triangular, which implies that the comultiplication is not braided. To understand the way in which the braiding appears in conformal field theory we analyze the decomposition of the tensor product into irreducible components. As the action of the symmetry algebra on the tensor product depends on two parameters, an intertwiner mapping the tensor product into an irreducible representation will in general have some dependence on these parameters as well. It turns out, that if one requires this intertwiner to transform naturally under scaling and translation, it will in general not be single valued, but exhibit the usual braiding. This seems to indicate that the braiding is no direct property of the symmetry algebra, but only arises once one tries to decompose the tensor product in a consistent way. In particular, we believe that this indicates, that the quantum group relevant for conformal field theory does not "sit inside" the symmetry algebra.

Finally, in section 4, we use the general framework to find all the restrictions for the fusion rules of the WZW- and the minimal models. Our method follows in spirit [6] and [2], but is quite different in flavour, as it is completely algebraic. In particular, having proved general properties of the tensor product, we can give a precise meaning to the intuitive argument of [2], which enables us to derive the general restrictions for the fusion rules of the minimal models from one special case. To prove that these conditions are also sufficient, we indicate how to show — using the methods of [1], [4], [10 — that the given representations are actually contained in the tensor product. This establishes that our definition of fusion agrees with the 
usual one.

\section{Comultiplication in CFT}

By analyzing conformal field theory we derive the comultiplication of the symmetry algebra, which gives rise to the vector space tensor product. We demonstrate the method explicitly for the WZW-models and give the result for the minimal models. This method can easily be generalized to other conformal field theories, since we only use the $s u(1,1)$-properties of the chiral algebra. (We shall pursue this in a forthcoming paper [5].)

One way to think about fusion is that it keeps track of the conformal families which occur in the operator product expansion of two vertex operators. To obtain the operator product expansion of two vertex operators one has to bring - at least heuristically - the two operators close together and determine the action of the symmetry algebra in the limit, where the two points coincide. In general this limit is singular and the expansion in the difference of the two insertion points contains fractional powers, reflecting the braid group statistics of the vertex operators. To avoid performing this limit one may alternatively consider the limit in which the holomorphic field (whose action on the fused state we want to determine) tends to infinity for fixed insertion points of the two vertex operators. Then one has to calculate the contour integral (over a large contour $C$, i. e. a contour encircling the two insertion points)

$$
\oint_{C} d w w^{m} J^{a}(w) V(\psi, \zeta) V(\chi, z) \Omega
$$

where $V(\psi, \zeta)$ and $V(\chi, z)$ denote the two vertex operators, $\Omega$ is the vacuum, $J^{a}(w)$ the current field in the symmetry algebra and $|\zeta|>|z|$ in order to guarantee the existence of the operator product. Assuming that the vertex operator and the current field are local with respect to each other one can apply the methods of [8] to obtain the operator product expansion of the two fields

$$
J^{a}(w) V(\psi, \zeta)=\sum_{l \in \mathbb{Z}}(w-\zeta)^{l-1} V\left(J_{-l}^{a} \psi, \zeta\right) .
$$

(If $\psi$ belongs to the dense subspace of finite energy states, the right-hand-side contains only a pole of finite order.) Then one can use the usual contour deformation arguments to evaluate (2.1), as the integrand has only poles at $0, z$ and $\zeta$ and (2.2) 
determines the residues. However, if we did the calculation at this stage, we would get (for $m<0$ ) a contribution from $w=0$ of the form

$$
V(\psi, \zeta) V(\chi, z) J_{m}^{a} \Omega
$$

and this term would not allow an interpretation as a comultiplication. We can circumvent this problem in the following way. Considering the scalar product of the integrand of (2.1) with any vector $\varphi \in \mathcal{F}$, the dense subspace of $\mathcal{H}$ of finite energy vectors

$$
\left\langle\varphi, J^{a}(w) V(\psi, \zeta) V(\chi, z) \Omega\right\rangle
$$

we get a meromorphic function of $w$, whose poles are determined by (2.2). Thus subtracting these poles we get an entire function, namely

$$
\begin{aligned}
\left\langle\varphi, J^{a}(w) V(\psi, \zeta) V(\chi, z) \Omega\right\rangle & -\sum_{m=-\infty}^{0}(w-\zeta)^{m-1}\left\langle\varphi, V\left(J_{-m}^{a} \psi, \zeta\right) V(\chi, z) \Omega\right\rangle \\
& -\sum_{l=-\infty}^{0}(w-z)^{l-1}\left\langle\varphi, V(\psi, \zeta) V\left(J_{-l}^{a} \chi, z\right) \Omega\right\rangle .
\end{aligned}
$$

Using the operator product expansion of the holomorphic field $J^{a}(w)$ with the vertex operator at $z$ we can write this function as

$$
\sum_{l=1}^{\infty}(w-z)^{l-1}\left\langle\varphi, V(\psi, \zeta) V\left(J_{-l}^{a} \chi, z\right) \Omega\right\rangle-\sum_{m=-\infty}^{0}(w-\zeta)^{m-1}\left\langle\varphi, V\left(J_{-m}^{a} \psi, \zeta\right) V(\chi, z) \Omega\right\rangle .
$$

Rewriting (2.6) as a power series about $w=0$ (assuming that it makes sense for $w=0$, i. e. that $\zeta$ and $z$ are suitably chosen) the series will converge for all $w$, as (2.6) is entire. As the integrand in (2.1) is just this entire function plus the poles we have subtracted in (2.5), we can now use this power series to evaluate the contour integral, thereby obtaining only terms where $J_{m}^{a}$ either acts on $\psi$ or on $\chi$. We remark that the action of $J$ on the operator product is independent of the vector $\varphi$. We can thus interpret the formula as giving a comultiplication of the Kac-Moody algebra, namely

$$
\begin{aligned}
& \oint_{C} d w w^{m}\left\langle\varphi, J^{a}(w) V(\psi, \zeta) V(\chi, z) \Omega\right\rangle= \\
& \sum\left\langle\varphi, V\left(\Delta_{\zeta, z}^{(1)}\left(J_{m}^{a}\right) \psi, \zeta\right) V\left(\Delta_{\zeta, z}^{(2)}\left(J_{m}^{a}\right) \chi, z\right) \Omega\right\rangle,
\end{aligned}
$$


where we write $\Delta_{\zeta, z}(a) \in \mathcal{A} \otimes \mathcal{A}, a \in \mathcal{A}$ as

$$
\Delta_{\zeta, z}(a)=\sum \Delta_{\zeta, z}^{(1)}(a) \otimes \Delta_{\zeta, z}^{(2)}(a) .
$$

We calculate

$$
\begin{aligned}
\Delta_{\zeta, z}\left(J_{n}^{a}\right)= & \sum_{m=0}^{n}\left(\begin{array}{c}
n \\
m
\end{array}\right) \zeta^{n-m}\left(J_{m}^{a} \otimes \mathbb{1}\right) \\
\Delta_{\zeta, z}\left(J_{-n}^{a}\right)= & \sum_{l=0}^{n}\left(\begin{array}{c}
n \\
l
\end{array}\right) z^{n-l}\left(\begin{array}{c}
n+m-1 \\
m
\end{array}\right)(-1)^{m} \zeta^{-(n+m)}\left(J_{m}^{a} \otimes \mathbb{1}\right) \\
& +\sum_{l=n}^{\infty}\left(\begin{array}{c}
l-1 \\
n-1
\end{array}\right)(-z)^{l-n}\left(\mathbb{1} \otimes J_{-l}^{a}\right),
\end{aligned}
$$

where in (2.9) $n \geq 0$ and in (2.10) $n \geq 1$.

Similarly, we can use the operator product expansion of the energy-momentum tensor with a vertex operator, e. g. as given in [8]

$$
T(w) V(\psi, \zeta)=\sum_{l \in \mathbb{Z}}(w-\zeta)^{l-2} V\left(L_{-l} \psi, \zeta\right)
$$

to derive the comultiplication for the Virasoro algebra, thereby obtaining

$$
\begin{array}{r}
\Delta_{\zeta, z}\left(L_{n}\right)=\sum_{m=-1}^{n}\left(\begin{array}{c}
n+1 \\
m+1
\end{array}\right) \zeta^{n-m}\left(L_{m} \otimes \mathbb{1}\right)+\sum_{l=-1}^{n}\left(\begin{array}{c}
n+1 \\
l+1
\end{array}\right) z^{n-l}\left(\mathbb{1} \otimes L_{l}\right) \\
\Delta_{\zeta, z}\left(L_{-n}\right)=\sum_{m=-1}^{\infty}\left(\begin{array}{c}
n+m-1 \\
m+1
\end{array}\right)(-1)^{m+1} \zeta^{-(n+m)}\left(L_{m} \otimes \mathbb{1}\right) \\
+\sum_{l=n}^{\infty}\left(\begin{array}{c}
l-2 \\
n-2
\end{array}\right)(-z)^{l-n}\left(\mathbb{1} \otimes L_{-l}\right),
\end{array}
$$

where in (2.12) $n \geq-1$ and in (2.13) $n \geq 2$. These formulae are generalizations of the comultiplication formulae given in [12], to which they reduce if we set $z=0$.

Formally the sums converge for $|\zeta|>1$ and $|z|<1$. If we restrict ourselves to a suitable dense subset of the tensor product (containing all tensor products of finite energy vectors) the comultiplication is well-defined for (a suitable subset of) $|z|<1$ and $\zeta \in \mathbb{C} \backslash\{0\}$. 
The representation induced by the comultiplication is a highest weight representation in the sense that for given highest weight vectors $\psi$ and $\chi$

$$
\Psi:=e^{-\zeta L_{-1}} \psi \otimes e^{-z L_{-1}} \chi
$$

is a highest weight vector for the action of $\Delta_{\zeta, z}$, i. e. $\Delta_{\zeta, z}\left(J_{n}^{a}\right) \Psi=0$ for $n>0$ and similarly for the Virasoro case. (This follows from the proof of (3.2) below.) However, $\Psi$ does not belong to the domain of definition for the comultiplication, as the action of $\Delta_{\zeta, z}\left(J_{-n}^{a}\right)$ is not well-defined on $\Psi$. We shall not discuss these problems in this paper.

To prove the comultiplication-property of these formulae, we have to check, that

$$
\left[\Delta_{\zeta . z}(a), \Delta_{\zeta . z}(b)\right]=\Delta_{\zeta . z}([a, b]) \quad \text { for all } a, b \in \mathcal{A},
$$

where $\mathcal{A}$ is the symmetry algebra, i. e. the Kac-Moody- or the Virasoro algebra, respectively. As this is quite technical we have banned an outline of the proof into appendix A.

The comultiplication formulae can be thought of as being the mode expansion of operator products involving a vertex operator and a holomorphic field. From this point of view the comultiplication-property reflects the fact, that the subalgebra of holomorphic fields is closed (and associative). However, as we derive these formulae form a well-defined conformal field theory, they will satisfy further relations reflecting various other properties of the theory. Some of these properties will be automatically satisfied on the level of the algebra (e. g. the comultiplication-property), however, as we shall see in the following, there are also relations, which do not hold true on the level of the algebra and which have to be implemented on the level of representations in order to maintain the whole information of conformal field theory.

\section{The ring-like tensor product}

The comultiplication formulae $(2.9-10,2.12-13)$ we have derived from conformal field theory are asymmetric in the sense that they treat the two algebras in the tensor product on a different footing. This is quite unsatisfactory, as one expects on general grounds that the symmetry algebra possesses a quasitriangular-like structure, reflecting the locality of the theory. 
To understand how to resolve this problem, let us recall, how we derived the comultiplication. (In the following we restrict ourselves to the case of the WZWmodels; obviously similar statements hold for the minimal models.) Assuming that $|\zeta|>|z|$ in order to guarantee the existence of the operator product, we used the operator product expansion of the holomorphic field at $w$ with the vertex operator at $z$ to get an expansion for the entire function (2.5), which could then be integrated. The comultiplication we obtained was well-defined for $|z|<1$ and $\zeta \in \mathbb{C} \backslash\{0\}$, if we restricted ourselves to the action on tensor products of finite energy vectors. However, we could have carried through the whole program with the rôles of $z$ and $\zeta$ interchanged, thereby getting instead of (2.10)

$$
\begin{aligned}
\widetilde{\Delta}_{\zeta, z}\left(J_{-n}^{a}\right)=\sum_{m=n}^{\infty}\left(\begin{array}{c}
m-1 \\
n-1
\end{array}\right) & (-\zeta)^{m-n}\left(J_{-m}^{a} \otimes \mathbb{1}\right) \\
& +\sum_{l=0}^{\infty}\left(\begin{array}{c}
n+l-1 \\
l
\end{array}\right)(-1)^{l} z^{-(n+l)}\left(\mathbb{1} \otimes J_{l}^{a}\right) .
\end{aligned}
$$

Again this formula is well-defined for $|\zeta|<1$ and $z \in \mathbb{C} \backslash\{0\}$ if we restrict ourselves to the action on tensor products of finite energy vectors. Thus in particular, for $z, \zeta \in\{u \in \mathbb{C}|| u \mid<1, u \neq 0\}$ the two formulae (2.10) and (3.1) should agree when restricted to this subset of vectors. (In fact, as we can see from (A.8) and (A.9), the two formulae are just different power series expansions of one analytic function.) However, this relation is not true on the level of algebras, and in order to maintain the whole information of conformal field theory we have to impose it on the level of representations. This is most naturally done in a way which closely resembles Richard Borcherds [3] proposal to regard fusion in conformal field theory as a ring-like tensor product of modules of the "quantum ring" of (holomorphic) fields. Namely, instead of defining the tensor product to be the usual (vector space) tensor product of representations, we define it to be the quotient of this vector space by all relations guaranteeing the equality of (2.10) and (3.1). This space is then the "true" tensor product of conformal field theory. The action of the symmetry algebra on it is given by either of the two comultiplication formulae.

The next step is to determine various general properties of this tensor product. Firstly we observe, that for $|u|<|\zeta|$ we have

$$
\left(e^{u L_{-1}} \otimes e^{v L_{-1}}\right) \circ \Delta_{\zeta+u, z+v} \circ\left(e^{-u L_{-1}} \otimes e^{-v L_{-1}}\right)=\Delta_{\zeta, z}
$$

and similarly for $|v|<|z|$

$$
\left(e^{u L_{-1}} \otimes e^{v L_{-1}}\right) \circ \widetilde{\Delta}_{\zeta+u, z+v} \circ\left(e^{-u L_{-1}} \otimes e^{-v L_{-1}}\right)=\widetilde{\Delta}_{\zeta, z} .
$$


The proof of (3.2), resp. (3.3) is just another standard manipulation of sums, once one observes that

$$
e^{u L_{-1}} J_{m}^{a} e^{-u L_{-1}}= \begin{cases}\sum_{l=0}^{m}\left(\begin{array}{c}
m \\
l
\end{array}\right)(-u)^{m-l} J_{l}^{a} & \text { if } m \geq 0 \\
\sum_{l=-m}^{\infty}\left(\begin{array}{c}
l-1 \\
-m-1
\end{array}\right) u^{l+m} J_{-l}^{a} & \text { if } m \leq-1,\end{cases}
$$

for $u$ sufficiently small.

(3.2) and (3.3) imply that the tensor products corresponding to different values of the parameters are equivalent, as we can define an intertwiner $U$, well-defined on the quotient

$$
\begin{gathered}
U: \mathcal{H}_{1} \otimes_{\zeta, z} \mathcal{H}_{2} \rightarrow \mathcal{H}_{1} \otimes_{\zeta+u, z+v} \mathcal{H}_{2} \\
\left(\psi \otimes_{\zeta, z} \chi\right) \mapsto\left(e^{-u L_{-1}} \psi \otimes_{\zeta+u, z+v} e^{-v L_{-1}} \chi\right),
\end{gathered}
$$

such that

$$
\begin{aligned}
& \Delta_{\zeta, z}=U^{-1} \circ \Delta_{\zeta+u, z+v} \circ U \\
& \widetilde{\Delta}_{\zeta, z}=U^{-1} \circ \widetilde{\Delta}_{\zeta+u, z+v} \circ U .
\end{aligned}
$$

Strictly speaking this is only true for those values of $\zeta, z, u$ and $v$ for which (3.2) and (3.3) make sense. However, as we may apply to the conformal nature of the theory, i. e. that the comultiplication must depend analytically on the parameters $\zeta$ and $z$, it is for example sufficient to prove the fusion rules for open neighbourhoods of suitably chosen values and thus, because of the above, only for one pair $(\zeta, z)$. (We will use this argument in the derivation of the fusion rules of the minimal models.)

As the operator product of the underlying conformal field theory is associative we expect the comultiplication to be coassociative. In fact, for suitable $\zeta_{1}, \zeta_{2}, z$ and $w$ we have

$$
\begin{aligned}
\left(\Delta_{\zeta_{2}-w, \zeta_{1}-w} \otimes \mathbb{1}\right) \circ \Delta_{w, z} & =\left(\mathbb{1} \otimes \Delta_{\zeta_{1}-w, z-w}\right) \circ \Delta_{\zeta_{2}, w} \\
\left(\Delta_{\zeta_{2}-w, \zeta_{1}-w} \otimes \mathbb{1}\right) \circ \widetilde{\Delta}_{w, z} & =\left(\mathbb{1} \otimes \widetilde{\Delta}_{\zeta_{1}-w, z-w}\right) \circ \Delta_{\zeta_{2}, w} \\
\left(\widetilde{\Delta}_{\zeta_{2}-w, \zeta_{1}-w} \otimes \mathbb{1}\right) \circ \widetilde{\Delta}_{w, z} & =\left(\mathbb{1} \otimes \Delta_{\zeta_{1}-w, z-w}\right) \circ \widetilde{\Delta}_{\zeta_{2}, w} \\
\left(\widetilde{\Delta}_{\zeta_{2}-w, \zeta_{1}-w} \otimes \mathbb{1}\right) \circ \Delta_{w, z} & =\left(\mathbb{1} \otimes \Delta_{\zeta_{1}-w, z-w}\right) \circ \Delta_{\zeta_{2}, w} \\
\left(\widetilde{\Delta}_{\zeta_{2}-w, \zeta_{1}-w} \otimes \mathbb{1}\right) \circ \widetilde{\Delta}_{w, z} & =\left(\mathbb{1} \otimes \widetilde{\Delta}_{\zeta_{1}-w, z-w}\right) \circ \widetilde{\Delta}_{\zeta_{2}, w},
\end{aligned}
$$

where $\widetilde{\Delta}$ denotes the comultiplication $(2.9,3.1)$ and $\Delta$ the original one $(2.9,2.10)$. The proof of these equations is again straight forward; for the convenience of the reader we have included the proof of one special case in appendix B. 
The above equations imply that the triple tensor product of conformal field theory is well-defined, as the subspace, by which we have to quotient the vector space tensor product, is independent of the bracketing. In addition, the action of the symmetry algebra on this tensor product is independent of the bracketing if we choose the parameters according to (3.7). Thus the tensor product is associative up to equivalence, as tensor products corresponding to different values of the parameters are equivalent.

We remark that using the same methods as in section 2 we could have also determined the action of the symmetry algebra on products of three vertex operators. A straight forward calculation shows that the action (3.7) agrees with this action if the vertex operators are inserted at $\zeta_{2}, \zeta_{1}$ and $z$. (The different expressions in (3.7) correspond to which operator product expansion we choose in (2.6).) Thus fusion of $n$ representations really corresponds to taking successively $(n-1)$ tensor products.

Thirdly, the $L_{0}$ action of conformal field theory translates into

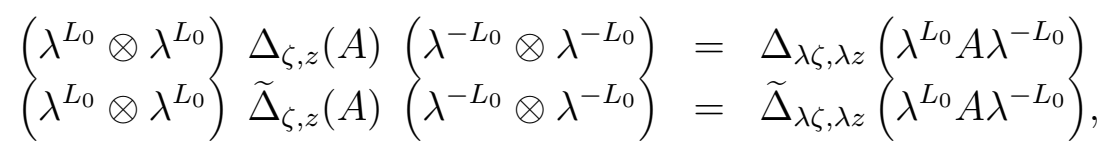

which can be checked very easily.

Finally, using the fact, that the two different comultiplications agree on these tensor products, we can find the universal $R$-matrix of the comultiplication, i. e. the operator $R(\zeta, z)$ in $\mathcal{A} \otimes \mathcal{A}$, which satisfies on all tensor products

$$
R(\zeta, z) \circ \Delta_{\zeta, z} \circ R(\zeta, z)^{-1}=\tau \circ \Delta_{\zeta, z}
$$

where $\tau$ is the twist-map, interchanging the two factors of the tensor product. In fact, using (3.2), one can easily show that $R(\zeta, z)$ is given by

$$
R(\zeta, z)=e^{(\zeta-z) L_{-1}} \otimes e^{(z-\zeta) L_{-1}}
$$

where we assume, that $\zeta$ and $z$ are suitably chosen to ensure convergence. At first sight (3.10) might seem quite surprising, as the $R$-matrix is triangular, i. e.

$$
\tau\left(R(\zeta, z)^{-1}\right)=R(\zeta, z)
$$

which implies, that the comultiplication is not braided. However, recalling the definition of the comultiplication from conformal field theory (2.7), we can easily 
understand, why this has to be true: $\Delta_{\zeta, z}^{(i)}\left(J_{m}^{a}\right)$ is just a Kac-Moody generator (or $\mathbb{1}$ ) and the monodromy properties of the scalar product depend only on the conformal families, hence the monodromy of the right and left hand side can only be equal, if $\Delta_{\zeta, z}$ has trivial monodromy, which is precisely (3.11). (Another way of understanding (3.11) is, that it reflects the fact that the action of the holomorphic fields on the vertex operators is local.) We would like to point out that this does not imply that the corresponding tensor category is not braided. For our comultiplication is not braided, but also not truly coassociative, as we have (3.7)

$$
\begin{aligned}
& \left(e^{\zeta_{1} L_{-1}} \otimes \mathbb{1} \otimes \mathbb{1}\right)\left(\left(\Delta_{\zeta_{2}, \zeta_{1}} \otimes \mathbb{1}\right) \circ \Delta_{\zeta_{1}, z}\right)\left(e^{-\zeta_{1} L_{-1}} \otimes \mathbb{1} \otimes \mathbb{1}\right) \\
& =\left(\mathbb{1} \otimes \mathbb{1} \otimes e^{\zeta_{1} L_{-1}}\right)\left(\left(\mathbb{1} \otimes \Delta_{\zeta_{1}, z}\right) \circ \Delta_{\zeta_{2}, \zeta_{1}}\right)\left(\mathbb{1} \otimes \mathbb{1} \otimes e^{-\zeta_{1} L_{-1}}\right) .
\end{aligned}
$$

(In fact, choosing all parameters to be the same, i. e. $\zeta=z \neq 0$, the comultiplication is symmetric and quasi-coassociative.)

To obtain some better understanding of the rôle of the braiding in conformal field theory let us next analyze the decomposition of the tensor product into irreducible components. Suppose the representation $\mathcal{H}_{3}$ is contained in the tensor product of two representations $\mathcal{H}_{i} \otimes_{\zeta, z} \mathcal{H}_{2}$, where the action of the symmetry algebra $\mathcal{A}$ is given by $\Delta_{\zeta, z}$. Then there exists an intertwiner for the action of the symmetry algebra

$$
\pi_{\zeta, z}: \mathcal{H}_{1} \otimes_{\zeta, z} \mathcal{H}_{2} \rightarrow \mathcal{H}_{3}
$$

i. e. a vector space homomorphism satisfying

$$
\pi_{\zeta, z} \circ \Delta_{\zeta, z}(a)=a \circ \pi_{\zeta, z}
$$

for all $a \in \mathcal{A}$, and a vector $\psi_{3} \in \mathcal{H}_{3}$, such that the scalar product

$$
F(\zeta, z)=\left\langle\psi_{3}, \pi_{\zeta, z}\left(\psi_{1} \otimes_{\zeta, z} \psi_{2}\right)\right\rangle
$$

does not vanish for all $\psi_{i} \in \mathcal{H}_{i}, i=1,2$. By Schur's Lemma, the intertwiner $\pi_{\zeta . z}$ is only determined up to a scalar (assuming no multiplicities, for simplicity). However, because of (3.2) and (3.8) there is a natural choice to relate the intertwiners for different values of $\zeta$ and $z$, as we can choose

$$
\pi_{\zeta-u, z-u}=e^{-u L_{-1}} \circ \pi_{\zeta, z}
$$


and

$$
\pi_{\lambda \zeta, \lambda z}=\lambda^{L_{0}} \circ \pi_{\zeta, z} \circ\left(\lambda^{-L_{0}} \otimes \lambda^{-L_{0}}\right),
$$

where in the second equation it is understood, that a choice for the logarithm of $\lambda$ has been made. This fixes the intertwiner up to an overall phase factor, if we allow for multivaluedness. If $\psi_{3} \in \mathcal{H}_{3}$ is now a Virasoro highest weight vector, then the above two equations imply that

$$
F(\zeta, z)=C\left(\psi_{1}, \psi_{2}, \psi_{3}\right)(\zeta-z)^{\Delta_{3}-\Delta_{1}-\Delta_{2}}
$$

where $L_{0} \psi_{i}=\Delta_{i} \psi_{i}, i=1,2,3$. Thus the scalar product, which corresponds to the three point function of conformal field theory, exhibits the expected braiding. However, the braiding does not seem to be a direct property of the symmetry algebra; it rather appears as we try to decompose the tensor product in a consistent way.

\section{Fusion rules}

In this section we want to show, how to derive the fusion rules of the WZW-and the minimal models from the above definition of fusion. We derive the restrictions for the possible fusions following essentially [6] and [2], but using an entirely algebraic language. To prove that these restrictions are sufficient in the case of the minimal models, we refer to the proofs of [1], [4], which can be easily adapted to the present situation.

Let us first consider the WZW-models, where the symmetry algebra is the affine Lie algebra $\hat{g}$ corresponding to the Lie algebra $g$ at level $x=2 k / \psi^{2}$ ( $\psi$ is any long root of $g$ and $k$ is the central parameter). The irreducible unitary positive energy representations of this affine Lie algebra for fixed level $x$ are labeled by the highest weight representations of the Lie algebra $g$, where in order for a representation of $g$ to be a highest weight representation of $\hat{g}$ the corresponding weight $\lambda$ must satisfy $|\langle\lambda, \beta\rangle| \leq k$ for all $\operatorname{roots} \beta$ [6], []].

The Kac-Moody representation $\mathcal{H}_{3}$ is contained in the tensor product of two $\hat{g}$-representations $\mathcal{H}_{1} \otimes_{\zeta, z} \mathcal{H}_{2}$, if there exists an intertwiner for the action of the Kac-Moody algebra $\pi_{\zeta, z}: \mathcal{H}_{1} \otimes_{\zeta, z} \mathcal{H}_{2} \rightarrow \mathcal{H}_{3}$, and a vector $\phi$ in the highest weight representation of $\mathcal{H}_{3}$, such that the scalar product $\left\langle\phi, \pi_{\zeta, z}\left(\psi_{1} \otimes_{\zeta, z} \psi_{2}\right)\right\rangle$ does not vanish for all highest weight vectors $\psi_{i}, i=1,2$. (In the limit $\zeta, z \rightarrow 0$ the righthand sides of the scalar products become the highest weight vectors of the tensor product - cf. the discussion following (2.14). However, we do not have to take 
this limit, as the scalar products either vanish identically or are non-zero for all $\zeta \neq z$, because of (3.18).) We can immediately observe — by considering the comultiplication (2.9) for $n=0$ - that a $\hat{g}$-representation will only be contained in the $\hat{g}$-tensor product of two $\hat{g}$-representations, if the corresponding highest weight representation is contained in the $g$-tensor product of the corresponding highest weight representations. To obtain further restrictions we observe - following [6], [7] - that the three generators

$$
J_{+1}^{-\theta} \quad J_{-1}^{\theta} \quad \frac{2}{\theta^{2}}\left(k-\theta \cdot H_{0}\right)
$$

satisfy the relations of an $s u(2)$-subalgebra, where $H_{0}$ denotes the elements of a Cartan subalgebra of $g$ and $J^{\theta}$ is a step operator corresponding to a root $\theta$ of g. Now, suppose that $\phi \in \mathcal{H}_{3}$ is a highest weight vector with weight $\phi$. Writing $M=\frac{2}{\theta^{2}}(k-\phi \cdot \theta)$ the above implies

$$
\left(J_{-1}^{\theta}\right)^{M+1} \phi=0
$$

as $J_{+1}^{-\theta} \phi=0$. Hence for all tensor products of highest weight vectors $\psi_{i} \in \mathcal{H}_{i}, i=1,2$ we have (in the following we suppress the subscripts of the tensor products)

$$
\begin{aligned}
0 & =\left\langle\left(J_{-1}^{\theta}\right)^{M+1} \phi, \pi_{\zeta, z}\left(\psi_{1} \otimes \psi_{2}\right)\right\rangle \\
& =\left\langle\phi, \pi_{\zeta, z}\left(\Delta_{\zeta, z}\left(J_{+1}^{-\theta}\right)^{M+1}\left(\psi_{1} \otimes \psi_{2}\right)\right)\right\rangle \\
& =\sum_{l=0}^{M+1}\left(\begin{array}{c}
M+1 \\
l
\end{array}\right) \zeta^{l} z^{M+1-l}\left\langle\phi, \pi_{\zeta, z}\left(\left(J_{0}^{-\theta}\right)^{l} \psi_{1} \otimes\left(J_{0}^{-\theta}\right)^{M+1-l} \psi_{2}\right)\right\rangle .
\end{aligned}
$$

All summands have to vanish separately, as we can vary $\zeta$ and $z$ independently and the $(\zeta, z)$ dependence of all scalar products is the same, as a consequence of (3.18). Writing $\phi_{1}=\left(J_{0}^{-\theta}\right)^{l_{1}} \psi_{1}$ and $\phi_{2}=\left(J_{0}^{-\theta}\right)^{M+1-l} \psi_{2}$ we recover [6, (3.49)], from which one can derive the "depth rule" of Gepner and Witten [6].

Next, we turn to deriving the fusion rules for the minimal models. Following the notation of [2] the primary fields $\psi_{(m, n)}$ of the theory are parametrized by two positive integers $m$ and $n$, giving the conformal weights of the corresponding fields via the equation

$$
\Delta_{(m, n)}=\Delta_{0}+\left(\frac{1}{2} \alpha_{+} m+\frac{1}{2} \alpha_{-} n\right)^{2}
$$


where

$$
\begin{gathered}
\Delta_{0}=\frac{1}{24}(c-1), \\
\alpha_{ \pm}=\frac{\sqrt{1-c} \pm \sqrt{25-c}}{\sqrt{24}}
\end{gathered}
$$

and $c$ is the central charge of the Virasoro algebra, $0 \leq c<1$. The primary fields of the theory are in one-to-one correspondence with a certain subset of the irreducible, positive energy representations of the Virasoro algebra and we denote the representation space corresponding to the field $\psi_{(m, n)}$ by $\mathcal{H}_{(m, n)}$. (The primary field $\psi_{(m, n)}$ is then a cyclic vector in $\mathcal{H}_{(m, n)}$ for the action of the Virasoro algebra.)

We restrict ourselves (without loss of generality) to analyzing the decomposition of the tensor product into irreducible representations for the case when $z=0$ and $\zeta \neq 0$, as the representations for different values of $z$ and $\zeta$ are equivalent, because of (3.2). Let us thus suppose that the representation $\mathcal{H}_{3}$ is contained in the tensor product of the two representations $\mathcal{H}_{(m, n)}$ and $\mathcal{H}_{(1,2)}$ (resp. $\left.\mathcal{H}_{(2,1)}\right)$. Then there exists an intertwiner (for the action of the Virasoro algebra) $\pi: \mathcal{H}_{(m, n)} \otimes \mathcal{H}_{(1,2)} \rightarrow \mathcal{H}_{\left(m^{\prime}, n^{\prime}\right)}$, such that the scalar product $\left\langle\psi_{\left(m^{\prime}, n^{\prime}\right)}, \pi\left(\psi_{(m, n)} \otimes \psi_{(1,2)}\right)\right\rangle$ does not vanish. Now, by definition, $\psi_{(1,2)}$ satisfies the equation ए

$$
\left(L_{-2}-\frac{3}{2\left(2 \Delta_{(1,2)}+1\right)} L_{-1}^{2}\right) \psi_{(1,2)}=0 .
$$

As $\psi_{\left(m^{\prime} n^{\prime}\right)}$ is a highest weight vector we thus obtain

$$
\begin{aligned}
0= & \left\langle\psi_{\left(m^{\prime}, n^{\prime}\right)}, \pi\left(\left(\Delta_{\zeta, 0}\left(L_{-2}\right)-\frac{3}{2\left(2 \Delta_{(1,2)}+1\right)} \Delta_{\zeta, 0}\left(L_{-1}\right)^{2}\right)\left(\psi_{(m, n)} \otimes \psi_{(1,2)}\right)\right)\right\rangle \\
= & \left\langle\psi_{\left(m^{\prime}, n^{\prime}\right)}, \pi\left(\left(\left(\mathbb{1} \otimes L_{-2}\right)-\frac{3}{2\left(2 \Delta_{(1,2)}+1\right)}\left(\mathbb{1} \otimes L_{-1}^{2}\right)\right)\left(\psi_{(m, n)} \otimes \psi_{(1,2)}\right)\right)\right\rangle \\
& +\left\langle\psi_{\left(m^{\prime}, n^{\prime}\right)}, \pi\left(\left(\zeta^{-1}\left(L_{-1} \otimes \mathbb{1}\right)-\zeta^{-2}\left(L_{0} \otimes \mathbb{1}\right)\right.\right.\right. \\
& \left.\left.\left.-\frac{3}{2\left(2 \Delta_{(1,2)}+1\right)}\left(2\left(L_{-1} \otimes L_{-1}\right)+\left(L_{-1}^{2} \otimes \mathbb{1}\right)\right)\right)\left(\psi_{(m, n)} \otimes \psi_{(1,2)}\right)\right)\right\rangle \\
= & \left\langle\psi_{\left(m^{\prime}, n^{\prime}\right)}, \pi\left(\left(\zeta^{-1}\left(L_{-1} \otimes \mathbb{1}\right)-\zeta^{-2} \Delta_{(m, n)}\right.\right.\right. \\
& \left.\left.\left.-\frac{3}{2\left(2 \Delta_{(1,2)}+1\right)}\left(2\left(L_{-1} \otimes L_{-1}\right)+\left(L_{-1}^{2} \otimes \mathbb{1}\right)\right)\right)\left(\psi_{(m, n)} \otimes \psi_{(1,2)}\right)\right)\right\rangle .
\end{aligned}
$$

\footnotetext{
${ }^{1}$ In formula (5.2) of [2] the coefficient of $L_{-1}^{2}$ has the wrong sign.
} 
To calculate the terms containing $L_{-1}$ we insert $L_{0}$ into the scalar product, using $\Delta_{\zeta, 0}\left(L_{0}\right)=\left(L_{0} \otimes \mathbb{1}\right)+\zeta\left(L_{-1} \otimes \mathbb{1}\right)+\left(\mathbb{1} \otimes L_{0}\right)$ to obtain

$$
\begin{aligned}
\Delta_{\left(m^{\prime}, n^{\prime}\right)}\langle & \left.\psi_{\left(m^{\prime}, n^{\prime}\right)}, \pi\left(\psi_{(m, n)} \otimes \psi_{(1,2)}\right)\right\rangle \\
= & \left\langle\psi_{\left(m^{\prime}, n^{\prime}\right)}, \pi\left(\zeta\left(L_{-1} \otimes \mathbb{1}\right)\left(\psi_{(m, n)} \otimes \psi_{(1,2)}\right)\right)\right\rangle \\
& \quad+\left(\Delta_{(m, n)}+\Delta_{(1,2)}\right)\left\langle\psi_{\left(m^{\prime}, n^{\prime}\right)}, \pi\left(\psi_{(m, n)} \otimes \psi_{(1,2)}\right)\right\rangle
\end{aligned}
$$

and similarly, by inserting $L_{0}^{2}$, we get

$$
\begin{aligned}
& \kappa(\kappa-1)\left(\psi_{\left(m^{\prime}, n^{\prime}\right)}, \pi\left(\psi_{(m, n)} \otimes \psi_{(1,2)}\right)\right) \\
& \quad=\left\langle\psi_{\left(m^{\prime}, n^{\prime}\right)}, \pi\left(\left(\zeta^{2}\left(L_{-1}^{2} \otimes \mathbb{1}\right)\right)\left(\psi_{(m, n)} \otimes \psi_{(1,2)}\right)\right)\right\rangle,
\end{aligned}
$$

where $\kappa=\Delta_{\left(m^{\prime}, n^{\prime}\right)}-\Delta_{(m, n)}-\Delta_{(1,2)}$. Finally,

$$
\begin{aligned}
0= & \left\langle\psi_{\left(m^{\prime}, n^{\prime}\right)}, \pi\left(\Delta_{\zeta, 0}\left(L_{0}\right) \Delta_{\zeta, 0}\left(L_{-1}\right)\left(\psi_{(m, n)} \otimes \psi_{(1,2)}\right)\right)\right\rangle \\
= & \left(\Delta_{(m, n)}+\Delta_{(1,2)}+1\right)\left\langle\psi_{\left(m^{\prime}, n^{\prime}\right)}, \pi\left(\Delta_{\zeta, 0}\left(L_{-1}\right)\left(\psi_{(m, n)} \otimes \psi_{(1,2)}\right)\right)\right\rangle \\
& +\left\langle\psi_{\left(m^{\prime}, n^{\prime}\right)}, \pi\left(\zeta\left(L_{-1}^{2} \otimes \mathbb{1}\right)\left(\psi_{(m, n)} \otimes \psi_{(1,2)}\right)\right)\right\rangle \\
& +\left\langle\psi_{\left(m^{\prime}, n^{\prime}\right)} \pi\left(\zeta\left(L_{-1} \otimes L_{-1}\right)\left(\psi_{(m, n)} \otimes \psi_{(1,2)}\right)\right)\right\rangle \\
= & \zeta^{-1} \kappa(\kappa-1)\left\langle\psi_{\left(m^{\prime}, n^{\prime}\right)}, \pi\left(\left(\psi_{(m, n)} \otimes \psi_{(1,2)}\right)\right)\right\rangle \\
& +\left\langle\psi_{\left(m^{\prime}, n^{\prime}\right)}, \pi\left(\zeta\left(L_{-1} \otimes L_{-1}\right)\left(\psi_{(m, n)} \otimes \psi_{(1,2)}\right)\right)\right\rangle,
\end{aligned}
$$

which implies

$$
\begin{aligned}
\left\langle\psi_{\left(m^{\prime}, n^{\prime}\right)}, \pi\right. & \left.\left(\left(L_{-1} \otimes L_{-1}\right)\left(\psi_{(m, n)} \otimes \psi_{(1,2)}\right)\right)\right\rangle \\
& =-\zeta^{-2} \kappa(\kappa-1)\left\langle\psi_{\left(m^{\prime}, n^{\prime}\right)}, \pi\left(\left(\psi_{(m, n)} \otimes \psi_{(1,2)}\right)\right)\right\rangle .
\end{aligned}
$$

Inserting (4.9), (4.10) and (4.12) into (4.8) we obtain

$$
0=\zeta^{-2}\left(\kappa-\Delta_{(m, n)}+\frac{3}{2\left(2 \Delta_{(1,2)}+1\right)} \kappa(\kappa-1)\right)\left\langle\psi_{\left(m^{\prime}, n^{\prime}\right)}, \pi\left(\psi_{(m, n)} \otimes \psi_{(1,2)}\right)\right\rangle .
$$

Hence, if the representation $\mathcal{H}_{\left(m^{\prime}, n^{\prime}\right)}$ is contained in the tensor product of $\mathcal{H}_{(m, n)}$ and $\mathcal{H}_{(1,2)}$, the bracket in (4.13) must vanish and $\Delta_{\left(m^{\prime}, n^{\prime}\right)}$ must satisfy equation (5.21) of 
[2] - thus giving the well-known restrictions for the fusion rules in the case where one of the fields is either $(1,2)$ or $(2,1)$

$$
\psi_{(1,2)} \otimes \psi_{(m, n)}=\left[\psi_{(m, n-1)}\right] \oplus\left[\psi_{(m, n+1)}\right]
$$

and

$$
\psi_{(2,1)} \otimes \psi_{(m, n)}=\left[\psi_{(m-1, n)}\right] \oplus\left[\psi_{(m+1, n)}\right]
$$

These equations imply in particular that the representation $\psi_{(m, n)}$ is contained in

$$
\left(\psi_{(2,1)}\right)^{\otimes(m-1)} \otimes\left(\psi_{(1,2)}\right)^{\otimes(n-1)} .
$$

Thus, any representation contained in $\psi_{\left(m_{1}, n_{1}\right)} \otimes \psi_{\left(m_{2}, n_{2}\right)}$ must also be contained in

$$
\left(\psi_{(2,1)}\right)^{\otimes\left(m_{1}-1\right)} \otimes\left(\psi_{(1,2)}\right)^{\otimes\left(n_{1}-1\right)} \otimes \psi_{\left(m_{2}, n_{2}\right)}
$$

and

$$
\left(\psi_{(2,1)}\right)^{\otimes\left(m_{2}-1\right)} \otimes\left(\psi_{(1,2)}\right)^{\otimes\left(n_{2}-1\right)} \otimes \psi_{\left(m_{1}, n_{1}\right)},
$$

where we have (implicitly) used the associativity and the symmetry of the tensor product. Using (4.14) and (4.15) we can derive from these two conditions the general restrictions for the fusion rules, namely

$$
\psi_{\left(m_{1}, n_{1}\right)} \otimes \psi_{\left(m_{2}, n_{2}\right)}=\sum_{k=\left|m_{1}-m_{2}\right|+1}^{m_{1}+m_{2}-1} \sum_{l=\left|n_{1}-n_{2}\right|+1}^{n_{1}+n_{2}-1}\left[\psi_{(k, l)}\right],
$$

where the variable $k(l)$ attains only every other value.

To prove that these conditions are also sufficient, we may apply the methods of [1], [4] or 10 to show, that the given irreducible representations are actually contained in the tensor product. In particular, our comultiplication is compatible with [1, (4.15)], which enables us to carry through the proof given there.

We remark, that the map $\mathcal{F}$ of [1] has non-trivial kernel. This reflects the fact that the true tensor product of conformal field theory is actually the quotient of the vector space tensor product, as described above. 


\section{Conclusions}

In this paper we have shown that fusion in conformal field theory can be understood as the ring-like tensor product of modules of the symmetry algebra. We have proved that the tensor product is associative and symmetric up to equivalence. We also determined explicitly the action of the symmetry algebra on these tensor products and derived the fusion rules of the WZW- and the minimal models.

We have thus established a framework within which determining the fusion rules is a well-posed algebraic problem. We would like to point out, that the above analysis can be generalized to a large class of conformal field theories [5], thus providing a powerful approach for determining the fusion rules for many conformal field theories.

To get a better understanding of the rôle of the braiding in conformal field theory we calculated the $R$-matrix of the comultiplication. It turned out, that the $R$-matrix is in fact triangular and that the braiding only occurs if one tries to decompose the tensor product into irreducible representations in a consistent way. This seems to indicate, that the quantum group relevant for conformal field theory does not "sit inside" the symmetry algebra. On the contrary, we rather believe, that the quantum group is connected to the fact, that in order to define consistently associative (chiral) vertex operators which only depend on the analytic parameter $z$, one is forced to retain some information of the action of the whole vertex operator on the "antiholomorphic representation spaces". In fact, for the case of the WZW-models, it turns out that it is sufficient to retain the action on the lowest-energy states, and these finite-dimensional vector spaces are isomorphic to the representation spaces of the corresponding quantum group $U_{q}(g)$. This structure was already anticipated in [11], however, a proper understanding is - in our opinion - still missing.

\section{A Proof of comultiplication}

Let us first consider the case of the Virasoro algebra. To prove the comultiplicationproperty one has to treat the different cases - corresponding to the different values of $n$ in (2.12), resp. (2.13) - separately. Writing out both sides of equation (2.15) 
the problem reduces to proving (e. g. for the case $a=L_{n}, b=L_{k}$ and $n, k \geq-1$ )

$$
\sum_{l=\max L-k,-1}^{\min L+1, n}\left(\begin{array}{c}
n+1 \\
l+1
\end{array}\right)\left(\begin{array}{c}
k+1 \\
L+1-l
\end{array}\right)(2 l-L)=(n-k)\left(\begin{array}{c}
n+k+1 \\
L+1
\end{array}\right) .
$$

To prove this we observe, that

$$
\sum_{m=-1}^{n}\left(\begin{array}{c}
n+1 \\
m+1
\end{array}\right) z^{n-m}=(z+1)^{n+1}
$$

and

$$
\begin{aligned}
\sum_{m=-1}^{n}\left(\begin{array}{c}
n+1 \\
m+1
\end{array}\right) z^{n-m} m & =\left.\frac{d}{d \varepsilon}(z+\varepsilon)^{n+1}\right|_{\varepsilon=1}-(z+1)^{n+1} \\
& =(n+1)(z+1)^{n}-(z+1)^{n+1}
\end{aligned}
$$

Hence we can calculate

$$
\sum_{l_{1}=-1}^{n} \sum_{l_{2}=-1}^{k}\left(\begin{array}{c}
n+1 \\
l_{1}+1
\end{array}\right) z^{n-l_{1}}\left(\begin{array}{c}
k+1 \\
l_{2}+1
\end{array}\right) z^{k-l_{2}}\left(l_{1}-l_{2}\right)=(n-k)(z+1)^{n+k+1} .
$$

On the other hand we can write the right-hand-side as

$$
(n-k) \sum_{L=-1}^{n+k}\left(\begin{array}{c}
n+k+1 \\
L+1
\end{array}\right) z^{n+k-L}
$$

and comparing the coefficients of the Cauchy product of the two sums in (A.4) with (A.5) we derive the desired formula (A.1).

The other cases can be dealt with similarly, once one observes that

$$
\begin{aligned}
\sum_{m=-1}^{\infty}\left(\begin{array}{c}
n+m-1 \\
m+1
\end{array}\right)(-1)^{m+1} z^{-(n+m)} & =\frac{(-1)^{n-1}}{(n-2) !} \frac{d^{n-2}}{d z^{n-2}} \sum_{m=-1}^{\infty}(-z)^{-(m+2)} \\
& =(z+1)^{1-n}
\end{aligned}
$$

and

$$
\begin{aligned}
\sum_{m=-1}^{\infty}\left(\begin{array}{c}
n+m-1 \\
m+1
\end{array}\right)(-1)^{m+1} z^{-(n+m)} m & =\left(-z \frac{d}{d z}-n\right)(z+1)^{1-n} \\
& =-(z+n)(z+1)^{-n}
\end{aligned}
$$


The proof in the case of the Kac-Moody algebra goes along similar lines. We would just like to point out that

$$
\begin{aligned}
\sum_{m=0}^{\infty}\left(\begin{array}{c}
n+m-1 \\
m
\end{array}\right)(-1)^{m} z^{-(n+m)} & =\frac{(-1)^{n}}{(n-1) !} \frac{d^{n-1}}{d z^{n-1}}\left(\frac{1}{1+z^{-1}}-1\right) \\
& =(z+1)^{-n}
\end{aligned}
$$

and

$$
\begin{aligned}
\sum_{m=n}^{\infty}\left(\begin{array}{c}
m-1 \\
n-1
\end{array}\right)(-z)^{m-n} & =\frac{(-1)^{n-1}}{(n-1) !} \frac{d^{n-1}}{d z^{n-1}} \sum_{m=1}^{\infty}(-z)^{m-1} \\
& =(z+1)^{-n}
\end{aligned}
$$

\section{B Coassociativity}

We give the proof of the coassociativity formula (3.7) for the case of the Virasoro algebra, when we apply both sides to $L_{-m}$ with $m>2$. There are three identities to check, corresponding to terms of the form $L_{k} \otimes \mathbb{1} \otimes \mathbb{1}, \quad \mathbb{1} \otimes L_{k} \otimes \mathbb{1}$ and $\mathbb{1} \otimes \mathbb{1} \otimes L_{k}$, respectively. The left-hand-side corresponding to the first term is

$$
\sum_{l=-1}^{\infty}\left(\begin{array}{c}
l+m-1 \\
m-2
\end{array}\right)(-1)^{l+1} \zeta_{1}^{-(m+l)} \sum_{k=-1}^{l}\left(\begin{array}{l}
l+1 \\
k+1
\end{array}\right)\left(\zeta_{2}-\zeta_{1}\right)^{l-k}\left(L_{k} \otimes \mathbb{1} \otimes \mathbb{1}\right),
$$

which can be resummed obtaining

$$
\sum_{k=-1}^{\infty}\left(\sum_{l=k}^{\infty}\left(\begin{array}{c}
l+m-1 \\
m-2
\end{array}\right)(-1)^{l+1}\left(\begin{array}{c}
l+1 \\
k+1
\end{array}\right)\left(\zeta_{2}-\zeta_{1}\right)^{l-k} \zeta_{1}^{-(m+l)}\right)\left(L_{k} \otimes \mathbb{1} \otimes \mathbb{1}\right) .
$$

The sum in the brackets can be calculated to be

$$
\begin{aligned}
& \frac{(k+m-1) !(-1)^{(k+1)}}{(k+1) !(m-2) !} \sum_{l=k}^{\infty} \frac{(l+m-1) !}{(m+k-1) !(l-k) !}(-1)^{l-k} \zeta_{1}^{-(m+l)}\left(\zeta_{2}-\zeta_{1}\right)^{l-k} \\
& =\left.\left(\begin{array}{c}
k+m-1 \\
k+1
\end{array}\right)(-1)^{k+1} \frac{(-1)^{m+k-1}}{(m+k-1) !} \frac{d^{m+k-1}}{d z_{1}^{m+k-1}} \sum_{l=0}^{\infty} z_{1}^{-(l+1)}\left(\zeta_{1}-\zeta_{2}\right)^{l}\right|_{z_{1}=\zeta_{1}} \\
& =\left(\begin{array}{c}
k+m-1 \\
k+1
\end{array}\right)(-1)^{k+1} \zeta_{2}^{-(m+k)},
\end{aligned}
$$


thus agreeing with the right-hand-side of (3.7) corresponding to the first term. The other terms can be dealt with similarly.

\section{Acknowledgements}

It is a pleasure to thank my PhD supervisor Peter Goddard for much advice and encouragement. I also want to thank Richard Borcherds for helpful remarks and M. Chu, H. Kausch, A. Kent, S. Majid, G. Segal and G. Watts for useful discussions.

I am grateful to Pembroke College, Cambridge, for a research studentship and to the Studienstiftung des deutschen Volkes for financial support.

\section{References}

[1] Bauer M., DiFrancesco P., Itzykson C., Zuber J.-B.: Covariant differential equations and singular vectors in Virasoro representations. Nucl. Phys. B 362, 515-562 (1991)

[2] Belavin A.A., Polyakov A.M., Zamolodchikov A.B.: Infinite Conformal Symmetry in two-dimensional quantum field theory. Nucl. Phys. B 241, 333-380 (1984)

[3] Borcherds R.: work in progress

[4] Feigin B.L., Fuchs D.B.: Cohomology of some nilpotent subalgebras of the Virasoro and Kac-Moody Lie algebras. J. Geom. Phys. 5, 209-235 (1988)

[5] Gaberdiel M.: work in progress

[6] Gepner D., Witten E.: String Theory on Group Manifolds. Nucl. Phys. B 278, 493-549 (1986)

[7] Goddard P.: Conformal Symmetry and its extensions. In: Simon B., Truman A., Davies I.M. (eds.) IX International Congress of Mathematical Physics. Proceedings, Swansea 1988, pp. 1-21. Bristol, New York: Adam Hilger 1989

[8] Goddard P.: Meromorphic conformal field theory. In: Kac V.G. (ed.) Infinite dimensional Lie Algebras and Lie Groups. Proceedings of CIRM-Luminy Conference 1988, p. 556. Singapore, New Jersey, Hong Kong: World Scientific 1989 
[9] Kazhdan D., Lusztig G.: Tensor structures arising from affine Lie Algebras I, II. preprints (1993)

[10] Kent A.: Fusion and the 3-point BPZ equations. (unpublished)

[11] Moore G., Reshetikhin N.: A comment on quantum group symmetry in conformal field theory. Nucl. Phys. B 328, 557-574 (1989)

[12] Moore G., Seiberg N.: Classical and Quantum Conformal Field Theory. Commun. Math. Phys. 123, 177-254 (1989)

[13] Segal G.: The Definition of Conformal Field Theory. Oxford preprint

[14] Wassermann A.: Loop groups and Operator Algebras. lecture easter term 1993, Cambridge 